including mutations in the 23S rRNA genes conferring varying levels of azithromycin resistance. Here, we report the emergence of a novel A to $G$ mutation at the 2058 nucleotide residue (A2058G) in the 23S rRNA genes, in two gonococcal isolates, that confers high-level resistance to azithromycin (HLAziR; $\geq 256 \mathrm{mg} / \mathrm{ml}$ ).

Methods The collection and antimicrobial susceptibility testing of $N$. gonorrhoeae isolates were performed as part of the Gonococcal Isolate Surveillance Project (GISP). Isolates with elevated minimum inhibitory concentration to azithromycin $(\geq$ $2 \mathrm{mg} / \mathrm{ml}$ ) were subjected to molecular analysis using Sanger PCR sequencing and/or whole genome sequencing analysis. Etest ${ }^{\circledR}$ was performed to confirm azithromycin susceptibility level and to determine the hetero-resistance phenotype (a concentration-dependent response to antibiotic) of the reported isolates.

Results Molecular analysis of GISP isolates from 2014-2018 revealed two isolates collected from two patients having the A2058G mutation in the 23S rRNA genes. One isolate had the HLAziR phenotype and A2058G mutations in all four $23 \mathrm{~S}$ rRNA. The second isolate had the A2058G mutation in three of the four alleles and displayed a hetero-resistance phenotype (azithromycin MIC ranging from $4 \mathrm{mg} / \mathrm{ml}$ to $\geq 256 \mathrm{mg} / \mathrm{ml}$ ). The wild-type allele was very conducive to A2058G conversion and resulted in a complete HLAziR phenotype. This mutational nucleotide conversion occurred in less than twenty hours after exposure to azithromycin using Etest ${ }^{\circledR}$.

Conclusion HLAziR in N. gonorrhoeae had largely been confined to isolates harboring a point mutation at nucleotide residue $\mathrm{A} 2059 \mathrm{G}$ of the $23 \mathrm{~S}$ rRNA genes. The newly discovered A2058G mutation further illuminates the genomic plasticity in N. gonorrhoeae when responding to antibiotic exposure and suggests a rapid recombination frequency between the $23 \mathrm{~S}$ rRNA alleles at this nucleotide residue.

Disclosure No significant relationships.

\section{P862 FEMALE SEX WORKERS AND THEIR ATTITUDE TOWARDS ORAL PRE-EXPOSURE PROPHYLAXIS}

${ }^{1}$ Tinashe Mudzviti', ${ }^{1}$ Anesu Dhliwayo, ${ }^{2}$ Byron Chingombe, ${ }^{3}$ Bernard Ngara, ${ }^{1}$ Tsitsi MoneraPenduka, ${ }^{1}$ Charles Maponga, ${ }^{4}$ Gene Morse. 'University of Zimbabwe, School of Pharmacy, Harare, Zimbabwe; ${ }^{2}$ Population Services International, Harare, Zimbabwe; ${ }^{3}$ University of Zimbabwe, Community Medicine, Harare, Zimbabwe; ${ }^{4}$ University at Buffalo, SUNY, Center for Integrated Global Biomedical Sciences, Buffalo, USA

10.1136/sextrans-2019-sti.903

Background Pre-exposure prophylaxis (PrEP) provides protection to sexually active persons at risk of acquiring HIV. Eligible female sex workers (FSWs) are a key population in which oral PrEP is indicated. The aim of this study was to evaluate knowledge levels of oral PrEP and the likelihood of its use among FSWs.

Methods A cross-sectional study in HIV uninfected FSWs was conducted. Interviews assessing awareness and intention to use PrEP were conducted initially. A description of PrEP as an HIV prevention strategy would be given after assessing awareness. Relative importance index was used to assess levels of knowledge, likelihood and barriers to PrEP use. A bivariate logistic regression model was utilized to identify predictors of PrEP use.
Results One hundred and thirty-one FSWs with a median age of 25 years (IQR: 21 - 31) participated. Most participants were single (78\%), 10\% being married, and $11 \%$ being either divorced or widowed. FSWs reported a median 5 (IQR: 3 6) daily sexual partners. Fifty-three (40\%) participants reported having at least one encounter of unprotected casual sexual intercourse within the preceding three months. Only 71 (54\%) participants had ever heard about PrEP. Of the FSWs that had heard about PrEP, 46 (35\%) had adequate knowledge on its use. A total of $102(78 \%)$ of the participants revealed that they would be willing to always use oral PrEP if it was provided to them for free. Likelihood of PrEP use increased among participants who had unprotected sex in the last 3 months $(r=0.0448, p=0.026)$. Participants that were more knowledge about PrEP had an increased likelihood for PrEP use $(r=0.21, p=0.0153)$.

Conclusion Knowledge of PrEP among FSWs in Zimbabwe was low. To increase uptake of PrEP as an HIV prevention strategy there will be need to further sensitize FSWs on this intervention.

Disclosure No significant relationships.

\section{P863 TRANSGENDER WOMEN IN LEBANON: A QUALITATIVE STUDY TO UNDERSTAND HIV/STIS RISK}

${ }^{1}$ Ismael Maatouk*, ${ }^{2}$ Rusi Jaspal. 'Clemenceau Medical Center, Dermatology-ST/S, Beirut, Lebanon; ${ }^{2}$ De Montfort University, Faculty of Life and Health Sciences, Leicester, UK

\subsection{6/sextrans-2019-sti.904}

Background In the Middle East and North Africa region, there is a scarcity of studies that focus on transgender individuals. Not only this group is regarded as one of the most at risk for HIV infection, but the burden of social stigma in this area of the world regarding transgender persons is very high. A single study by Kaplan et al. that focused on HIV related risk and resilience among 10 transgender women (TW) in Lebanon reported $0 \%$ HIV rate. The aim of this study was to characterize TW, a hard-to-reach population, and to understand HIV/STIs risky behavior in a sample in Lebanon.

Methods Interviews were conducted with 18 Lebanese TW using an STI clinic and checkpoint in Lebanon. Data were analyzed using Qualitative Thematic Analysis.

Results Three principal themes were raised: social issues; stigma, discrimination/trauma and mental health issues; healthcare access.High risky behavior as condomless sexual intercourses were mentioned due to social and family rejections, difficult to access healthcare services, and stigma with discrimination. However, according to the findings, access to HIV/ STIs services was considered better when compared to other health services.

Conclusion This qualitative study investigated HIV/STIs risk behaviors of TW in Lebanon. Our findings about social and family issues, discrimination and stigma, health care access difficulty even from LGBT community should help local programs to better understand the TW population in Lebanon and their needs. Special services addressing TW in non-medical and medical issues, taking into consideration an overall comprehensive and multi-disciplinary intervention appears to be the most useful.

Disclosure No significant relationships. 